\title{
Coronavirus Anxiety Scale: Content Validity and Reliability of Arabic Version Samir El Sayed ${ }^{1}$, Doaa Shokry ${ }^{2}$, Sarah Gomaa ${ }^{3}$
}

${ }^{1}$ Department of Psychiatry, ${ }^{2}$ Department of Public Health, ${ }^{3}$ Department Psychiatry, Faculty of Medicine, Mansoura University Students' Hospital,Mansoura University, Mansoura, Egypt

*Corresponding author: Samir El Sayed, Mobile: (+20) 01025747441, E-Mail: shinnysky83@ gmail.com

\begin{abstract}
Background: Psychiatric manifestations of individuals impacted by the coronavirus pandemic have not been sufficiently investigated. It is very important to find a short valid and reliable scale for particularly assessing the anxiety manifestations during coronavirus disease -2019 (COVID-19) pandemic which is suitable in our culture leading to early intervention and management. Objectives: The current study aimed to validate the Arabic version of corona virus anxiety scale (CAS) and assess its reliability and translation into Arabic.

Patients and Methods: CAS has been translated into Arabic. Methodological design was used to investigate content validity index (CVI) reported by eleven expert jurors. The Arabic version was distributed among 700 participants who completed the scale. Intra class correlation coefficient (inter-rater and intra-rater agreement) and Cronbach's $\alpha$ reliability coefficients were addressed to investigate reliability. Results: The CVI Item for relevance ranged from 0.90 to 1.0 and for clarity from 0.71 to 1.0. All inter-rater and intra-rater correlation coefficients are positive and significant and ranges from 0.78 to 0.92 for inter-rater and from 0.88 to 0.96 for intra-rater. The inter-rater and intra-rater correlation coefficients of the total score were 0.83 and 0.92 , respectively. The total Cronbach's $\alpha$ of the total scale was 0.92 .

Conclusion: Corona virus anxiety scale (CAS) Arabic version based on the results is valid, reliable, stable, and its translation is convenient to the culture. It can be used for screening of anxiety manifestations concomitant with Covid 19 pandemic.
\end{abstract}

Keywords: Arabic, Corona Virus Anxiety, Pandemic, Reliability.

\section{INTRODUCTION}

On 31 December 2019, a unique viral pneumonia emerging in Wuhan, China was declared to the World Health Organization ${ }^{(\mathbf{1})}$. As of 23 March 2020, this novel coronavirus (COVID-19) speeded across the entire world, infecting more than 294,110 people in 187 countries and killing 12,944 individuals ${ }^{(2)}$.

Xiang et al. ${ }^{(3)}$ noted although there has been a considerable effort to investigate people with the coronavirus infection, assessing the psychiatric status of people impacted by this pandemic was not fair enough.

Balaratnasingam and Janca (4) highlighted during the pandemic of infectious diseases, there are often elaborated high levels of stress and anxiety that lead to disturbance of the behavioral and psychological wellbeing of the individuals.

Liu et al. ${ }^{(5)}$ mentioned in a recent, large survey of people highly vulnerable to the coronavirus infection, including Chinese medical workers, the prevalence rate of traumatic stress was at an alarming $73.4 \%$, depression was at $50.7 \%$, generalized anxiety was at $44.7 \%$, and insomnia was at $36.1 \%$.

Previous researches were done during the infectious outbreak found clear relationship between pandemic related anxiety and high stress levels, anxiety, post-traumatic stress disorder, contamination thoughts, and suicidal ideations ${ }^{(6)}$.

The World Health Organization has declared that such curfew/social distancing measurements might result in people becoming more anxious, angry, stressed, agitated, and withdrawn ${ }^{(7)}$.

Santini et al. ${ }^{(8)}$ said that a crucial risk factor for both anxiety and depression is perceived isolation.

Anxiety is considered the most common mental disorders and defined as a persistent feeling of worry, apprehension fear, or nervousness, also approximately 1 in 10 people living in the UK suffer from an anxiety related disorder ${ }^{(9)}$. The coronavirus anxiety scale (CAS) is a 5-item mental health screener implemented to help the health care professionals to find the possible cases of COVID 19-related handicapping anxiety by an efficient and effective way ${ }^{(10)}$. Asmundson and Taylor (11) released the terminology of "coronaphobia" which referred to corona virus anxiety in their emerging research. The data regarding this CAS has been presented previously by Lee ${ }^{(\mathbf{1 0})}$.

The current study aimed to validate the Arabic version of corona virus anxiety scale (CAS) and assess its reliability and translation into Arabic.

\section{PATIENTS AND METHODS}

This study was conducted in Hayat National Hospital, Psychiatry Department, Riyadh, Kingdom of Saudi Arabia. Data were gathered from $10^{\text {th }}$ of June to $25^{\text {th }}$ of June 2020.

This current methodological (validation) design is a cross sectional study with a longitudinal component for measuring the reliability and content validity of the Arabic version of coronavirus anxiety scale (CAS). 
A convenience sample of 700 participants of medical staff, nursing staff, patients attended to different clinics and their caregivers, all of them completed the scale.

All participants of both sexes, with age range from 18-60 years, can read and write to complete the scale, and were not diagnosed with COVID 19 during the last 2 weeks. Those who have major psychiatric disorders or received psychotropic medications were excluded from the study. Coronavirus anxiety scale was developed by Lee ${ }^{(\mathbf{1 0})}$, which is used as a short screening scale for assessment of anxiety manifestations during the COVID 19 pandemic. Each item has the cognitive items like worry, behavioral like sociooccupational dysfunctioning, affective like anxiety and physiological including sleep problems; tonic paralysis. Each item was ranked on a 5-point Likert-type scale to evaluate the frequency of the manifestations, ranging from 0 (not at all) to 4 (nearly every day) over the last 2 weeks. This format of the scale was generated on the DSM-5's anxiety symptom measure, adult form of self-reported one (12). The translation of the CAS followed the scientific recommendations of adaptation of health related scales ${ }^{\mathbf{( 1 3 )}}$. The process was carried out in 5 stages: forward translation, synthesis of the translated copy, back translation, expert committee, and test of the prefinal one.

The scale was translated from English into Arabic by two independent translators whose native language is Arabic (T1 and T2).

The Arabic version of the scale (T1-2) was translated back into English by other independent qualified translators, then 2 back translated copied were generated (B1 and B2).

The scale was cross-culturally examined by an expert committee consisted of 4 translators involved in the forward and back-process, an expert of research methodology and a professor of psychiatry. The committee was requested to revise the generated material (T1.T2, T12, BT1, and BT2) and the original copy. At the end, researchers generated a dependable prefinal Arabic copy of studied scale.

The prefinal one was evaluated on thirty participants (not to be a part of the study sample) who were informed to complete the Arabic scale. After that, cognitive debriefing process was done, each individual was asked about every component of the scale and his answer. To examine the content validity, concept of 11 expert staff was obtained. Arabic version of the scale was evaluated for clarity, relevance, and translation of the scale. The Arabic version was given to 700 participants. Intra class correlation coefficient ICC (inter-rater and intra rater agreement) and Cronbach's $\alpha$ reliability coefficients were addressed to assess the reliability.

Ethical approval and consent to participate:

Institutional research board (IRB) approval was taken to carry out this study, which was in accordance of Declaration of Helsinki. Permission of reproduction of the scale and translation into Arabic was achieved from the generator of the scale. Informed verbal consent was taken from all those who took part in this study after giving them the detailed information about the aim of the study and its steps. Participants were informed about the confidentiality of their information and their ability to stop participation in this study at any time without giving any causes.

\section{Statistical analysis}

Data were analyzed using the Statistical Package for the Social Sciences (SPSS) program for Windows Standard version 24 (2018 by IBM United States of America USA, The English modality was used). The normality of data was first tested with one-sample Kolmogorov-Smirnov test. CVIs were calculated for each item and each expert. Pearson's correlation coefficient ( $r$ ) was used to measure inter-rater and intrarater correlation. Cronbach's $\alpha$ was calculated to measure the internal consistency between items. Qualitative data were described using number and percent. Continuous variables were presented as mean \pm SD and two groups were compared by t-test while ANOVA test was used to compare more than 2 groups. The results were considered significant when $(\mathrm{p} \leq 0.05)$.

\section{RESULTS}

Table (1) shows the sociodemographic data of the studied group.

Table (1): Sociodemographic data among the studied group

\begin{tabular}{|ll|}
\hline Sociodemographic data & $\begin{array}{l}\text { The studied group } \\
\text { (n=700) }\end{array}$ \\
\hline Age/ years & \\
Mean \pm SD & $\mathbf{3 7 . 3 1} \pm \mathbf{1 0 . 4 3}$ \\
$<30$ y & $198(28.3 \%)$ \\
$30-40$ y & $195(27.9 \%)$ \\
$40-50$ y & $203(29.0 \%)$ \\
$>50$ y & $104(14.9 \%)$ \\
\hline Gender & \\
Male & $395(56.4 \%)$ \\
Female & $305(43.6 \%)$ \\
\hline Marital status & \\
Married & $456(65.1 \%)$ \\
Single & $244(34.9 \%)$ \\
\hline Education & \\
Preparatory & $141(20.1 \%)$ \\
Secondary & $231(33.0 \%)$ \\
University & $328(46.9 \%)$ \\
\hline Occupation & \\
Worker & $406(58.0 \%)$ \\
Non worker & $294(42.0 \%)$ \\
\hline Residence & \\
Rural & $484(69.1 \%)$ \\
Urban & $216(30.9 \%)$ \\
\hline Smoking & \\
Smokers & $358(51.1 \%)$ \\
Non smokers & $342(48.9 \%)$ \\
\hline
\end{tabular}


Table (2) shows results distribution of CAS components.

Table (2): Results distribution of coronavirus anxiety scale components among participants

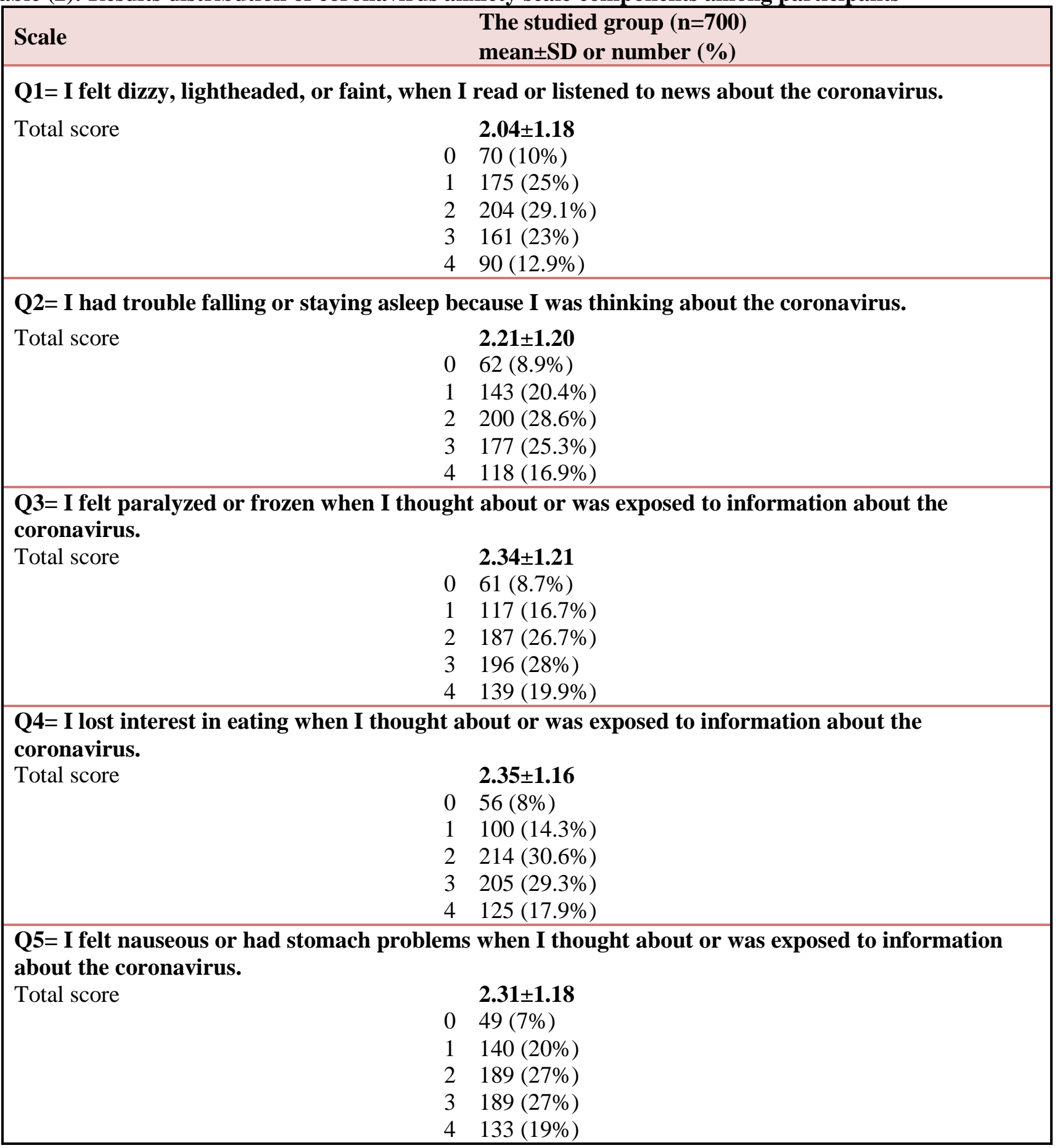

\section{Q=question}

Table (3) shows statistically significant difference in groups of participants according to age and Q3 of CAS. Also there is a statistically significant groups in married versus single participants regarding Q1, 3 and 5 of CAS. Level of education of the participants has a statistically significant difference with Q4 of CAS and a statistically significant difference between employment of the participants and Q5 of CAS. 
Table (3): Correlation between sociodemographic data of the participants and components of corona virus anxiety scale CAS

\begin{tabular}{|c|c|c|c|c|c|}
\hline $\begin{array}{c}\text { Socio- } \\
\text { demographic } \\
\text { data }\end{array}$ & $\begin{array}{c}\text { Q1= I felt } \\
\text { dizzy, } \\
\text { lightheaded, or } \\
\text { faint, when I } \\
\text { read or } \\
\text { listened to } \\
\text { news about the } \\
\text { coronavirus }\end{array}$ & $\begin{array}{c}\text { Q2 =I had } \\
\text { trouble } \\
\text { falling or } \\
\text { staying asleep } \\
\text { because I was } \\
\text { thinking } \\
\text { about the } \\
\text { coronavirus }\end{array}$ & $\begin{array}{c}\text { Q3= I felt } \\
\text { paralyzed or } \\
\text { frozen when I } \\
\text { thought } \\
\text { about or was } \\
\text { exposed to } \\
\text { information } \\
\text { about the } \\
\text { coronavirus }\end{array}$ & $\begin{array}{c}\text { Q4 = I lost } \\
\text { interest in } \\
\text { eating when I } \\
\text { thought } \\
\text { about or was } \\
\text { exposed to } \\
\text { information } \\
\text { about the } \\
\text { coronavirus }\end{array}$ & $\begin{array}{c}\text { Q5= I felt } \\
\text { nauseous or had } \\
\text { stomach problems } \\
\text { when I thought } \\
\text { about or was } \\
\text { exposed to } \\
\text { information about } \\
\text { the coronavirus }\end{array}$ \\
\hline \multicolumn{6}{|l|}{ Age/ years } \\
\hline$<30 \mathrm{y}$ & $2.04 \pm 1.14$ & $2.15 \pm 1.24$ & $2.07 \pm 1.21^{\mathrm{abc}}$ & $2.30 \pm 1.08$ & $2.38 \pm 1.18$ \\
\hline $30-40 y$ & $2.0 \pm 1.23$ & $2.29 \pm 1.20$ & $2.36 \pm 1.20^{\mathrm{a}}$ & $2.43 \pm 1.21$ & $2.36 \pm 1.21$ \\
\hline $40-50 \mathrm{y}$ & $2.03 \pm 1.19$ & $2.22 \pm 1.16$ & $2.49 \pm 1.13^{b}$ & $2.21 \pm 1.2$ & $2.23 \pm 1.17$ \\
\hline$>50 \mathrm{y}$ & $2.13 \pm 1.12$ & $2.15 \pm 1.18$ & $2.48 \pm 1.32^{\mathrm{c}}$ & $2.55 \pm 1.08$ & $2.23 \pm 1.2$ \\
\hline$P$ value & 0.855 & 0.660 & $0.002 *$ & 0.061 & 0.454 \\
\hline \multicolumn{6}{|l|}{ Gender } \\
\hline Male & $2.05 \pm 1.16$ & $2.18 \pm 1.18$ & $2.36 \pm 1.17$ & $2.33 \pm 1.14$ & $2.33 \pm 1.20$ \\
\hline Female & $2.02 \pm 1.21$ & $2.25 \pm 1.22$ & $2.30 \pm 1.27$ & $2.37 \pm 1.18$ & $2.29 \pm 1.17$ \\
\hline$P$ value & 0.731 & 0.432 & 0.515 & 0.641 & 0.675 \\
\hline \multicolumn{6}{|l|}{ Marital status } \\
\hline Married & $2.01 \pm 1.18$ & $2.15 \pm 1.18$ & $2.25 \pm 1.23$ & $2.34 \pm 1.17$ & $2.27 \pm 1.21$ \\
\hline Single & $2.27 \pm 1.16$ & $2.33 \pm 1.24$ & $2.48 \pm 1.19$ & $2.43 \pm 1.18$ & $2.47 \pm 1.19$ \\
\hline$P$ value & $0.006^{*}$ & 0.07 & $0.017 *$ & 0.358 & $0.039 *$ \\
\hline \multicolumn{6}{|l|}{ Education } \\
\hline Preparatory & $1.94 \pm 1.16$ & $2.33 \pm 1.21$ & $2.16 \pm 1.14$ & $2.22 \pm 1.24^{\mathrm{a}}$ & $2.35 \pm 1.18$ \\
\hline Secondary & $2.0 \pm 1.21$ & $2.12 \pm 1.18$ & $2.33 \pm 1.18$ & $2.51 \pm 1.09^{a b}$ & $2.44 \pm 1.17$ \\
\hline University & $2.1 \pm 1.16$ & $2.22 \pm 1.21$ & $2.41 \pm 1.26$ & $2.29 \pm 1.16^{\mathrm{b}}$ & $2.20 \pm 1.19$ \\
\hline$P$ value & 0.365 & 0.254 & 0.108 & $0.028^{*}$ & 0.061 \\
\hline \multicolumn{6}{|l|}{ Occupation } \\
\hline Worker & $2.09 \pm 1.18$ & $2.19 \pm 1.19$ & $2.39 \pm 1.18$ & $2.32 \pm 1.18$ & $2.39 \pm 1.16$ \\
\hline Non worker & $1.96 \pm 1.17$ & $2.23 \pm 1.21$ & $2.25 \pm 1.26$ & $2.39 \pm 1.13$ & $2.20 \pm 1.22$ \\
\hline$P$ value & 0.137 & 0.625 & 0.136 & 0.432 & $0.045^{*}$ \\
\hline \multicolumn{6}{|l|}{ Residence } \\
\hline Rural & $2.01 \pm 1.18$ & $2.22 \pm 1.21$ & $2.35 \pm 1.22$ & $2.38 \pm 1.16$ & $2.31 \pm 1.22$ \\
\hline Urban & $2.09 \pm 1.18$ & $2.19 \pm 1.16$ & $2.30 \pm 1.22$ & $2.28 \pm 1.16$ & $2.31 \pm 1.12$ \\
\hline$P$ value & 0.407 & 0.783 & 0.614 & 0.325 & 0.947 \\
\hline \multicolumn{6}{|l|}{ Smoking } \\
\hline Smokers & $2.01 \pm 1.18$ & $2.19 \pm 1.16$ & $2.40 \pm 1.2$ & $2.35 \pm 1.14$ & $2.27 \pm 1.22$ \\
\hline Non smokers & $2.06 \pm 1.17$ & $2.23 \pm 1.23$ & $2.27 \pm 1.23$ & $2.34 \pm 1.18$ & $2.35 \pm 1.16$ \\
\hline P value & 0.596 & 0.630 & 0.156 & 0.911 & 0.410 \\
\hline
\end{tabular}

abc: similar letters indicate significant difference between groups

\section{DISCUSSION}

The aim of the study was to validate the psychometric properties of the Arabic version of coronavirus anxiety scale (CAS) as a brief screener for anxiety manifestations in coronavirus era, to our knowledge it is the first published psychopathological COVID-19 related scale that helped the health care professional to detect high levels of dysfunctional anxiety during the pandemic. The CAS is considered a brief mental scale, its diagnostic parameters $(90 \%$ sensitivity and $85 \%$ specificity) are comparable to different psychiatric screening tests. For example ${ }^{(14)}$, noted that the sensitivity (89\%) and specificity (82\%) values for the Generalized Anxiety Disorder 7 (GAD7), a popular tool of anxiety disorder symptoms, are slightly lower than those of the CAS.

Although the CAS items center on anxiety and coronavirus related situations, it is also targeting the somatic physical manifestations relating to the anxiety (e.g., illness anxiety disorder) ${ }^{(\mathbf{1 2})}$.

Presence of Arabic version of CAS as the first psychometric tool was particularly originated to help us in the Arabic countries to evaluate the anxiety 
manifestations during this critical period of COVID 19 pandemic.

In our current study the CVI Item for relevance ranged from 0.90 to 1.0 and for clarity from 0.71 to 1.0 . All inter-rater and intra-rater correlation coefficients are positive and significant and ranges from 0.78 to 0.92 for inter-rater and from 0.88 to 0.96 for intrarater. The inter-rater and intra-rater correlation coefficients of the total score were 0.83 and 0.92 , respectively. The total Cronbach's $\alpha$ of the total scale was 0.92 , which represented homogeneity between the items of the scale. Our study targeted to provide a short brief less time consuming Arabic scale for evaluation of anxiety in COVID 19 pandemic.

\section{CONCLUSION}

In conclusion, CAS Arabic version based on the results is valid, reliable, and stable, and its translation is convenient to the culture. More research is mandatory for assessment and management for those who have COVID 19 -related dysfunctional anxiety.

\section{REFERENCES}

1. World Health Organization (2020a): WHO Director-General's opening remarks at the media briefing on COVID-19.

https://www.who.int/dg/speeches/detail/who-directorgeneral-s-opening-remarks-at-the-media-briefing-oncovid-19---11-march-2020

2. World Health Organization (2020b): Coronavirus disease (COVID-19) outbreak situation. https://www.who.int/emergencies/diseases/novelcoronavirus-2019/situation-reports

3. Xiang Y, Zhao Y, Liu Z et al. (2020): The COVID-19 outbreak and psychiatric hospitals in China: managing challenges through mental health service reform. Int J Biol Sci., 16(10):1741-4.

4. Balaratnasingam S, Janca A (2006): Mass hysteria revisited. Curr Opin in Psych., 19:171-174.

5. Liu S, Yang L, Zhang C et al. (2020): Online mental health services in China during the COVID-19 outbreak. The Lanc Psych., 4: 17-18.

6. Wheaton M, Abramowitz J, Berman N et al. (2012): Cognitive Therapy and Research. Psychological predictors of anxiety in response to the H1N1 (swine flu) pandemic. Cogni Thera and Resea., 36(3): 210-218.

7. World Health Organization (2020): Depression. https://www.who.int/news-room/fact-sheets/ detail/depression .

8. Santini Z, Jose P, Cornwell E (2020): Social disconnectedness, perceived isolation, and symptoms of depression and anxiety among older Americans (NSHAP): a longitudinal mediation analysis. Lancet Pub Heal, 5: 6270 .

9. Mental Health UK (2020): What is anxiety? https://mentalhealth-uk.org/help-and-information/ conditions/anxiety-disorders/what-is-anxiety/

10. Lee S (2020): Coronavirus Anxiety Scale: A brief mental health screener for COVID-19 related anxiety. Death Stud., 44 (7): 393-401.

11. Asmundson G, Taylor S (2020): Coronaphobia: Fear and the 2019-nCoV outbreak. J. Anxie Disord., 70: 102196.

12. American Psychiatric Association (2013): Diagnostic and statistical manual of mental disorders (5th ed.). https://doi.org/10.1176/appi.books.9780890425596.

13. Beaton E, Bombardier C, Guillemin F et al. (2000): Guidelines for the process of cross cultural adaptation of self-report measures. Spin, 25:3186-91.

14. Spitzer R, Kroenke K, Williams J et al. (2006): A brief measure for assessing generalized anxiety disorder: The GAD-7. Archi of Inter Medi., 166(10), 1092-1097. 